\title{
Space-time evolution of electron-beam driven electron holes and their effects on the plasma
}

\author{
N. Singh \\ Department of Electrical and Computer Engineering, University of Alabama in Huntsville, Huntsville, AL 35899, USA
}

Received: 4 December 2001 - Revised: 22 March 2002 - Accepted: 25 March 2002

\begin{abstract}
We report here further results from the threedimensional particle-in-cell simulations of the electron-beam driven electron holes. We focus here on (i) the transformation of oscillatory waves driven by the electron-beam instability into electron holes, (ii) the continued evolution and propagation of electron holes after their formation, including merging of electron holes, and (iii) the effects of the evolution on the plasma density and ion velocity distribution function. We find that initially electron-beam modes with perpendicular wave numbers $k_{\perp}=0$ and as well as $k_{\perp} \neq 0$ are driven resonantly below the electron plasma frequency of the target plasma. The modes interact nonlinearly and modulate each other both in space and time, producing wave structures with finite perpendicular scale lengths. Nonlinear evolution of such wave structures generates the electron holes in the simulations. Initially, a large number of electron holes form in the plasma. Their merging yields continuously a decreasing number of electron holes. The propagation velocity of the electron holes evolves dynamically and is affected by their merging. At late times only a few electron holes are left in the simulation and they decay by emitting low-frequency electrostatic whistler waves just above the lower hybrid (LH) frequency $\omega_{\ell h}$. These waves, which are long structures parallel to the ambient magnetic field $\boldsymbol{B}_{0}$ and quite short transverse to $\boldsymbol{B}_{0}$, are associated with similar structures in the plasma density, producing density filaments. It turns out that electronbeam driven plasmas, in general, develop such filaments at some stage of the evolution of the beam-driven waves. In view of the excitation of the LH waves near $\omega_{\ell h}$, which could resonate with the ions, an analysis shows that it is possible to heat transversely the ions in a time scale of a few seconds in the auroral return current plasma, in which electron holes and transversely heated ions have been simultaneously observed.
\end{abstract}

\section{Introduction}

Electron holes are fast propagating positive potential pulses. In a magnetized plasma, they propagate along the ambient magnetic field $\boldsymbol{B}_{0}$. Although such structures have been

Correspondence to: N. Singh (singh@ece.uah.edu) known in plasma physics since the early simulations of nonlinear stage of the electron-electron (e-e) two-stream instability (e.g. see Berk and Roberts, 1967), they were recently detected by several satellites ranging from FAST in the auroral plasma (e.g. Ergun et al., 1998a, b), Polar in the distant magnetosphere (Mozer et al., 1997), Geotail in the magnetotail (Matsumotto et al., 1994) and Wind in the bowshock and the solar wind plasmas. Following their detection in space, there is a spurt of theoretical and simulation studies on the formation of multi-dimensional structures and the stability of electron holes (Omura et al., 1996, 1999, Goldman et al., 1999; Miyake et al., 1998; Oppenheim et al., 1999; Singh, 2000; Singh et al., 2001a, b; Muschietti et al., 1999, 2000). Before the detection and following the spurt of these theoretical studies, there have been experimental (Saieki et al., 1979), as well as theoretical studies (Schamell, 1982). The experimental results of Saieki et al. were simulated by Lynov et al. (1979) using a 1-D PIC code. Turikov (1984) studied the stability of electron holes. Formation and propagation of electron holes were studied by simulations of double layers (Hubbard and Joyce, 1979; Singh 1980, 1982). Singh and Schunk (1984) showed that electron holes are a common feature of the high-potential side of a double layer. This feature was further demonstrated by 1-D Vlasov simulations of double layers, and the properties of the electron holes were compared with those detected from FAST (Ergun et al., 1998a, b; Singh, 2000).

Recently, we have performed 3-D PIC simulations of electron-beam driven instabilities and found that for relatively slow beams the nonlinear stage of the instability-driven waves consists of electron holes, which could be long lasting, but ultimately decay by emitting low-frequency electrostatic whistler waves just above the lower hybrid frequency (Singh et al., 2000, 2001a, b). The purpose of this paper is to further study the following issues: (i) emergence of electron holes from oscillating waves generated by electron-beam driven instability, (ii) evolution and propagation of electron holes including merging when electron holes come in close proximity, and (iii) effects of the electron holes on the plasma including generation of low-frequency LH waves, associated density structure in the plasma, and transverse acceleration 

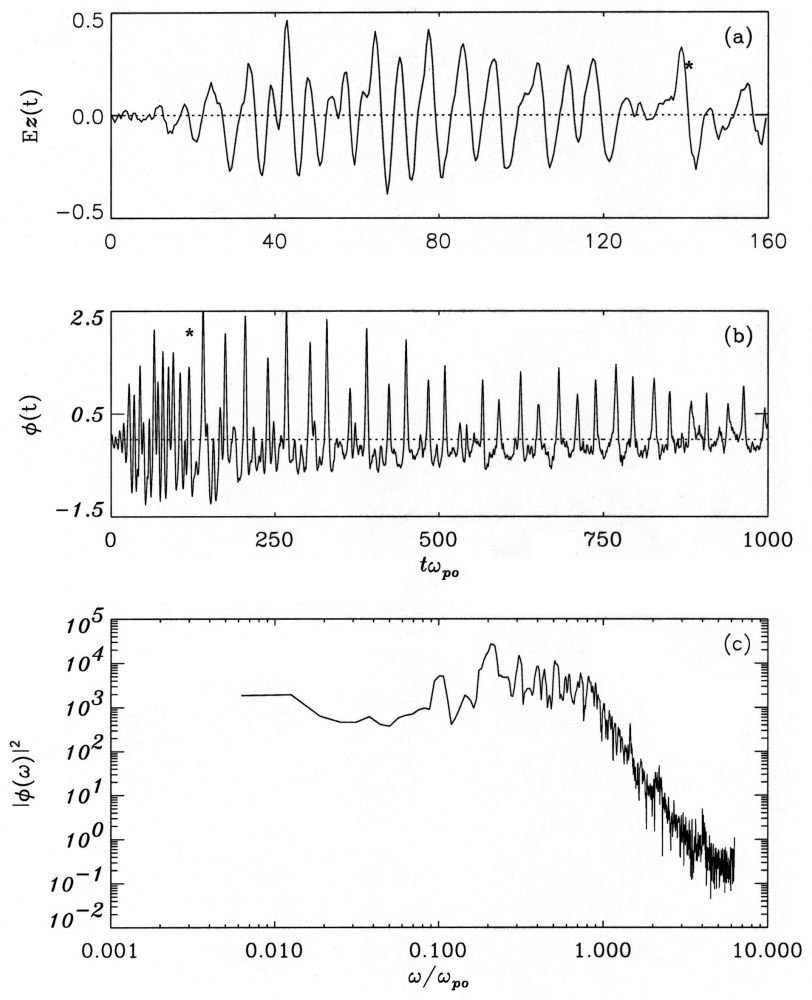

Fig. 1. Temporal evolution of the waves driven by the electron beam with $n_{b}=0.1 n_{o}$ and $V_{b}=4 V_{t e}$. (a) Early time evolution of the parallel field $E_{z}(32,32,9)$ leading to the formation of a bipolar solitary structure at $\bar{t} \sim 140$. Prior to this time the waves are oscillatory and are undergoing temporal modulation. (b) Temporal evolution of the potential $\phi(32,32,9)$ over the entire simulation time showing oscillatory waves being transformed into solitary pulses, which periodically appear at the point $(32,32,9)$. The pulse marked with the $*$ is the bipolar pulse in (a) near $\bar{t} \sim 140$. (c) Frequency spectrum of the data plotted in (b) showing a broad band spectrum.

of ions.

The rest of the paper is organized as follows. The simulation model is briefly described in Sect. 2. In Sect. 3, we describe the numerical results; the discussion here includes the formation of electron holes of finite transverse sizes, their propagation and merging. We also discuss here wave and plasma structure in the late stage of the evolution and transverse ion heating. The conclusion is given in Sect. 4.

\section{Simulation model}

The simulation model has been described previously by Singh et al. (2000, 2001a-c). Here we describe briefly the method, giving sufficient information to follow the rest of the paper. Simulations were preformed using a fully 3-D electrostatic parallel PIC code (Loo et al., 1999). Periodic boundary conditions on both particles and fields were used. We performed several simulations by varying (1) the parameters of the electron beam, (2) the size of the 3-D simulation volume given by $L_{x} \times L_{y} \times L_{z}$, and (3) the ratio of the
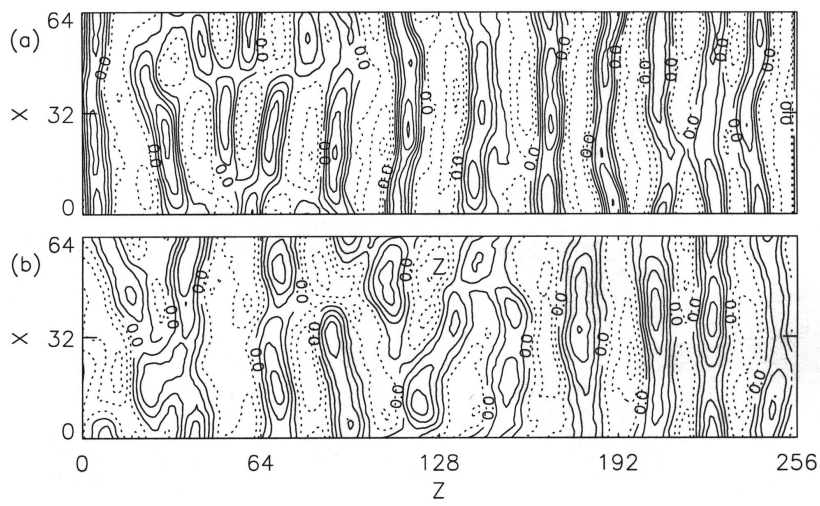

Fig. 2. Temporal evolution of the wave structure. Equipotential contours are plotted at (a) $\bar{t}=30$ and (b) $\bar{t}=50$ in the $x-z$ plane at $\bar{y}=32$. The contours with $\phi=0$ are labeled; other contours are $\Delta \phi=0.2$ apart. The wave structures consist of waves with $k_{\perp}=0$ as well as $k \neq 0$, where $k_{\perp}$ is the perpendicular wave number. Note the progressive breaking of the waves in the transverse direction $x$. A similar behavior occurs in the $y-z$ planes.

electron plasma $\left(\omega_{p e}\right)$ to cyclotron $\left(\Omega_{e}\right)$ frequency. We note that the $z$ direction is parallel to the ambient magnetic field $\boldsymbol{B}_{0}$, and that $x$ and $y$ are transverse to $\boldsymbol{B}_{0}$. In the simulations performed so far, the beam velocity along $z\left(\| \boldsymbol{B}_{0}\right)$ was assumed to be $V_{b}=4 V_{t e}$ or $8 V_{t e}$, where $V_{t e}$ is the initial electron thermal velocity. The initial electron and ion temperatures were assumed to be equal, i.e. $T_{e}=T_{i}=T_{o}$ and $V_{t e}=\left(k_{B} T_{o} / m_{e}\right)^{1 / 2}$, where $k_{B}$ is the Boltzmann constant and $m_{e}$ is the electron mass. Beam temperature was varied in different simulations, but as long as $V_{b} \gg V_{t e}>V_{t b}$, the beam thermal velocity, the actual value of $V_{t b}$ did not matter much. In the runs we used 36 pairs of electrons and ions in each cell of size $\lambda_{d o}^{3}$. In the simulations described here, the electron beam density $n_{b}$ was $10 \%, V_{b} / V_{t e}=4$ and $V_{t b} / V_{t e}=0.1$. For the larger velocity quoted above, longlasting electron-holes did not form, even though well-formed vortexes occurred in the phase space at very early times (Singh et al., 2001c). The simulations were performed for ion to electron mass ratio $M / m_{e}=1836, L_{x}=L_{y}=64 \lambda_{d o}$ and $L_{z}=256 \lambda_{d o}$. In our discussions, we have used the following normalizations and definitions: distance $\bar{x}=x / \lambda_{d o}$, time $\bar{t}=t \omega_{p o}$, velocity $\bar{V}=V / V_{t e}$, potential $\bar{\phi}=e \phi / k_{B} T_{o}$, electric field $\bar{E}=E / k_{B} T_{o} / e \lambda_{d o}$, and $\lambda_{d o}=V_{t e} / \omega_{p o}$, where $\omega_{p o}$ is the electron plasma frequency with the total plasma density $n_{o}$.

\section{Numerical results}

We have performed several simulations in which the electron magnetization $\left(\Omega_{e} / \omega_{p e}\right)$ was varied. We have reported previously the results from these simulations (Singh et al., 2000, 2001a, b). Here we study some specific issues in greater detail than in previously work; specifically, these issues deal with the formation and motion of electron holes and their mutual interaction. We also discuss the formation of density 
(a)

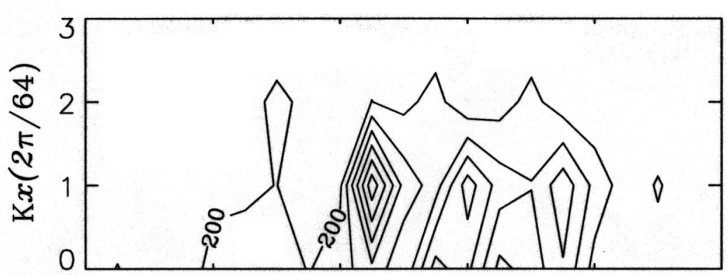

(b)

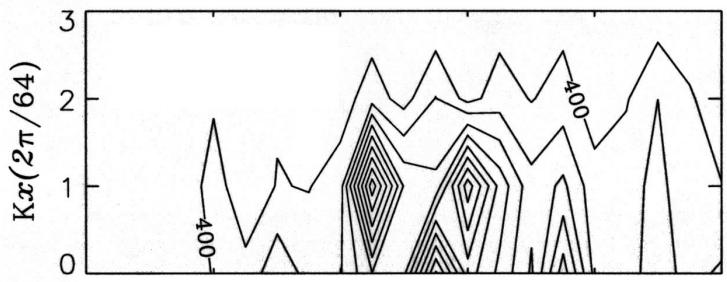

(c)

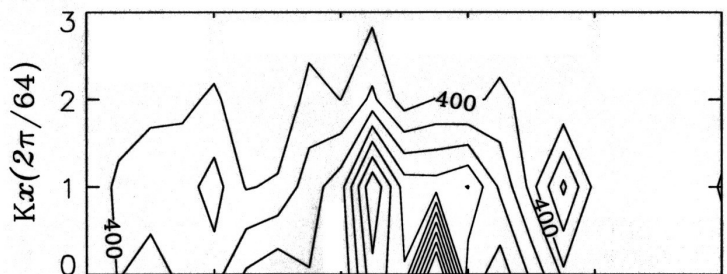

(d)

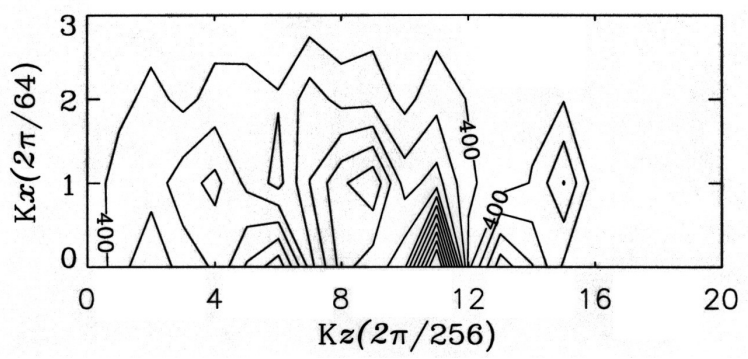

Fig. 3. Fourier spectrum of the wave structures like that shown in Figs. 2a and b at several times: (a) $\bar{t}=20$, (b) $\bar{t}=30$, (c) $\bar{t}=40$, and (d) $\bar{t}=50$. The vertical and horizontal axes are labeled with mode numbers $n$ and $m$, respectively. Contours are 100 units apart and show relative power. Note the dominance of wave power in modes with $6 \leq n \leq 16$ and $m<2$.

striations in association with the low-frequency (LF) electrostatic whistler-waves emitted by the electron holes as they decay. Furthermore, here we examine the transverse heating of ions; despite substantial perpendicular electric fields in the electron hole structures and also in the LF waves, we find that ion velocity distribution is affected only by the trapping in the waves without a significant transverse heating. Most of the discussion in this paper is based on a simulation with $\Omega_{e} / \omega_{p e}=2$. The effects of the variation in $\Omega_{e} / \omega_{p e}$ on the formation and stability of electron holes is discussed in Singh et al. (2001b).

\subsection{Early time behavior consisting of linear waves}

It is now commonly believed that the electron holes could emerge from high-frequency waves driven by the electronelectron (e-e) two-stream instability (e.g. see Omura et al., 1999; Goldman et al., 1999; Singh et al., 2000; Oppenheim et al., 1999). However, the process involved in the evolu- tion of the high-frequency (HF) waves into electron holes of finite transverse dimensions at a very early stage, as seen in our 3-D simulations, has not been fully explored. We explore here this issue as revealed by our simulations in combination with the linear analysis of the e-e instability. We examine the processes which occur successively after the electron beam is switched in a simulation with $\Omega_{e} / \omega_{p e}=2$. Figure 1a shows the temporal evolution of the parallel electric field $E_{z}$ over the early time interval showing (i) growth of the oscillations in $E_{z}(t)$, and (ii) modulation in the oscillation followed by the appearance of a bipolar pulse at about $\bar{t} \sim 140$. This bipolar pulse is a fast moving, positive pulse and it is a vortex structure in the $z-V_{z}$ phase space. Figure $1 \mathrm{~b}$ gives the time history of this pulse, as discussed later in more detail. Figure 1c shows the frequency spectrum of the time-series data in Fig. 1b. Note the broad spectrum associated with the high frequency waves generated by the electron beam and its evolution at later times.

The spatial structure of the evolving waves is shown in Figs. 2a and $\mathrm{b}$, in which the equipotential contours of the wave potential are plotted in the $x-z(\bar{y}=32)$ plane at $\bar{t}=30$ and 50. The structures in the $y-z$ plane are similar and are not shown here. These figures show that the wave structure consists of waves with perpendicular wave number $k_{\perp}$, as well as $k_{\perp} \neq 0$; the latter feature is revealed by the perpendicular modulations of the equipotential structures. We find that perpendicular modulations appear as the waves grow (Fig. 1a). In order to understand these features of the growing waves, we first examine the structures in the $k_{z}-k_{x}$ plane at times $\bar{t}=20-50$, as plotted in Figs. 3a-d. The plots are the FFTs of the potential structures in the $x-z$ planes. In Figs. 3a-d, the horizontal and vertical axes are labeled with mode numbers $n$ and $m$, respectively. The mode numbers are defined by $k_{z}=n 2 \pi / L_{z}$ and $k_{x}=m 2 \pi / L_{x}$. We see from Figs. 3a-d that from a very early time $\bar{t}=20$, the dominantly driven modes have $m=0$ and $m=1$ and $8<n<16$. Let us see if such modes are linearly driven by the beam with $n_{b}=0.1 n_{o}$ and $V_{b}=4 V_{t e}$ used in the simulation.

The electrostatic linear dispersion relation is given by

$k_{\perp}^{2} \varepsilon_{\perp}+k_{\|}^{2} \varepsilon_{\|}=0$,

where under the cold-plasma approximation

$\varepsilon_{\perp}=1-\frac{\omega_{p t}^{2}}{\omega^{2}-\Omega_{e}^{2}}-\frac{\omega_{p b}^{2}}{\left(\omega-k_{\|} V_{b}\right)^{2}-\Omega_{e}^{2}}$,

$\varepsilon_{\|}=1-\frac{\omega_{p t}^{2}}{\omega^{2}}-\frac{\omega_{p b}^{2}}{\left(\omega-k_{\|} V_{b}\right)^{2}}$,

$k_{\perp}\left(k_{\|}\right)$is the component of the wave vector $\boldsymbol{k}$ perpendicular (parallel) to $\boldsymbol{B}_{o}, \omega_{p t}\left(\omega_{p b}\right)$ is the target (beam) electron plasma frequency corresponding to the plasma density $n_{t}\left(n_{b}\right)$, so that $n_{t}+n_{b}=n_{o}$. We look for waves over $\omega_{\ell h}<\omega<\omega_{p t}$, where $\omega_{\ell h}$ is the lower hybrid frequency. For $\omega_{\ell h} \ll \omega<\omega_{p t} \ll \Omega_{e}$, Eq. (1) can be written as

$\alpha_{t} / \bar{\omega}^{2}+\alpha_{b} /\left(\bar{\omega}-k_{\|} V_{b} / \omega_{p o}\right)^{2} \cong 1+\frac{k_{\perp}^{2}}{k_{\|}^{2}}\left(1+\omega_{p o}^{2} / \Omega_{e}^{2}\right)$, 


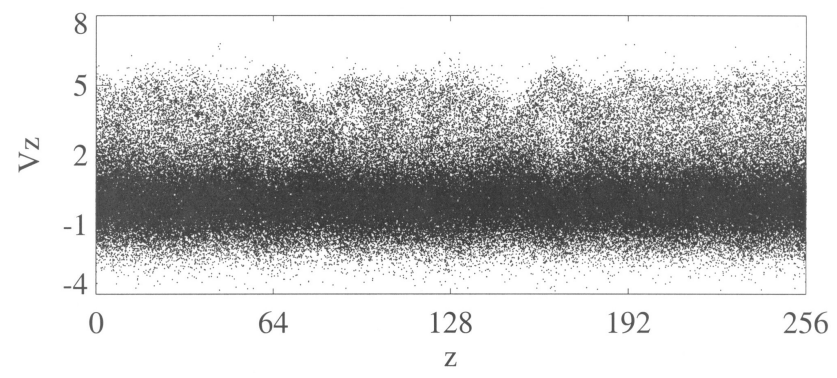

Fig. 4. Phase space structure in the $z-V_{z}$ plane at $\bar{t}=100$ showing vortex formation. Such vortexes are the signature of electron holes. The potential structure corresponding to this phase space is plotted in Fig. 5a.

where $\bar{\omega}=\omega / \omega_{p o}, \alpha_{t}=n_{t} / n_{o}$ and $\alpha_{b}=n_{b} / n_{o}$.

Performing a marginal stability analysis for the beam parameters $\alpha_{b}=0.1, \alpha_{1}=0.9$ and $V_{b}=4 V_{t e}$, we find that Eq. (4) shows roots of $\omega$ for $m<3$ and over a broad range of values of $n$. Table 1 lists the real $\left(\omega_{r}\right)$ and imaginary $(\gamma)$ parts of $\omega$ for several modes determined by $m, n$; we list here the values of $\omega_{r}$ and $\gamma$ as $\omega_{r} / \gamma$. The symbol $S$ represents stable modes. This table shows that significant growth rates are possible for $6 \leq n<14$ and $m \leq 3$, but the growth is the largest for $10 \tilde{<} n \tilde{<} 12$ and $m=0$, and the growth rate for $m=1$ is quite close to the growth rates for $m=0$. These features of the linear instability are well reflected in Figs. 3a-d, both the $m=0$ and $m=1$ modes seem to be equally strong for $6 \leq n \leq 14$. As these modes grow they interact nonlinearly with each other and thereby the spatial and temporal modulation of the waves occur. As the waves grow, the depth of modulation grows and the waves continue to break into segments, revealed by the wave structures in Figs. 2a-b.

As the processes of breaking the long $\lambda_{\perp}$ waves and their growth continue, the electron trapping occurs, forming vortexes in the phase-space. Figure 4 shows the vortex formation at $\bar{t}=100$. Corresponding to the vortexes in Fig. 4, the potential structure at $\bar{t}=100$ is shown in Fig. 5a.

\subsection{Space-time behavior of electron holes}

Figures 5a-e show the evolution of the potential structure in the $x-z$ plane at $\bar{y}=32$ and the corresponding evolution in the $y-z$ plane $(\bar{x}=32)$ is shown in Figs. $5 \mathrm{f}-\mathrm{j}$. Together, in these $x-z$ and $y-z$ planes, we show nearly all the electron hole structures in the volume of the simulated plasma, some of which are numbered. Some of the electron holes are common to the $x-z$ and $y-z$ planes, while others are seen only in one of the two planes shown. As shown in Fig. 1a, the oscillatory behavior of the waves continues until about $t \cong 140$, after which solitary behavior dominates. This later behavior is seen clearly from Fig. $1 \mathrm{~b}$ in which the time history of the potential at the point $\mathrm{P}_{1}(32,32,9)$ is plotted, showing quite regular appearances of solitary potential pulses. The bipolar electric field pulse at $t \cong 140$ in Fig. 1a corresponds to the passage of the positive potential pulse through the point $\mathrm{P}_{1}(32,32,9)$ marked with a star in Fig. 1b. This pulse and some others appear periodically at point $\mathrm{P}_{1}$, because our simulation system is periodic; the pulse exiting at $z=L_{z}$ reappears at $z=0$. In other words, the pulse train appearing in Fig. 1b represents an infinite chain of pulses. The frequency spectrum of this periodic chain of pulses, along with the initial oscillations for $\bar{t}<140$, is shown in Fig. 1c. The spectrum is quite broad band.

We now discuss the structural change in the wave pattern as the transition occurs from oscillatory to solitary behavior. The panels (a) and (f) in Fig. 5 show that at $\bar{t}=100$, the initially dominant modes have interacted nonlinearly, greatly modifying the potential distribution seen earlier at $\bar{t}=30$ and 50 in Figs. 2a and b. The waves have broken into shorter structures in the transverse direction and have developed strongly peaked features. The wave structure at $\bar{t}=100$ involves nearly equally strong negative and positive values of the peaks in $\phi(x, y, z)$ (also see Figs. 1a and b). Even though the waves are oscillatory, there are structured vortexes in the $z-V_{z}$ phase space, as we saw in Fig. 4. The vortexes correspond to the positive peaks in the potential distribution. Panels (b) and (g) for $\bar{t}=200$ show that the number of positive peaks in the potential distribution have diminished and at the same time the value of the negative potential is also diminished (also see Fig. 1b). Thus, the initially oscillatory potential distribution in the plasma has transformed into highly structured potential pulses, which are the electron holes. Some of the pulses are numbered for further reference. We note that nearly half of the structure of \# 1 is located near $z=0$, while the other half is near $z=256$. This is the effect of periodic boundary condition; likewise, \#4 and \# 5 also appear divided. Panels (b) and (g) show that \# 1 and \# 2 are common to both $\bar{x}=32$ and $\bar{y}=32$ planes. There are other electron holes in the $\bar{x}=32$ plane numbered as \# 6-9. The electron holes shown in panels (b) and (g) further evolve and the main feature of the evolution consists of the merging of the weaker electron holes with stronger ones, until about $\bar{t} \cong 500$. Panels (c)-(e) and (h)-(j) show that by the time $\bar{t}=500$ there are fewer structures as numbered.

We wish to highlight an interesting situation involving the merging of several electron holes. In Fig. $5 \mathrm{~g}$, we notice the presence of electron holes \# 1, 2, 6, 7, 8 and 9 at $\bar{t}=200$. We can see most of these structures at $\bar{t}=300$ and 400, and also at 500 in panels (h), (i,) and (j), respectively; 7 has merged with \# 6 as noted by $6 / 7$. Of course the surviving ones undergo some structural changes as they circulate around the simulation plasma with a velocity near $V_{H} \sim 4$. They also differ in their velocity somewhat. By the time $\bar{t}=500$, the electron holes \# 2, 8 in the $y-z$ plane and \#3, which earlier appeared only in the $x-z$ plane and now appears in $y-z$ plane $(x=32)$, are in close proximity to each other and they share some of their equipotential contours.

Like Figs. 5a-j, Figs. 6a-j show the evolutions in the $x-z$ and $y-z$ planes from $\bar{t}=600$ to 1000 . The merging at $\bar{t} \sim 500$ discussed above, continues as seen from Figs. 6a and f. Panel (a) shows that \#2 and 3 (see Fig. 5e) have 
Table 1. Real $\left(\omega_{r}\right)$ and imaginary $(\gamma)$ parts of $\omega$ for the modes $(m, n)$ are listed as $\omega_{r} / \gamma$

\begin{tabular}{cccccccccc}
\hline$m / n$ & 6 & 7 & 8 & 9 & 10 & 11 & 12 & 13 & 14 \\
\hline 0 & $0.50 / 0.18$ & $0.57 / 0.20$ & $0.63 / 0.23$ & $0.69 / 0.24$ & $0.76 / 0.26$ & $0.81 / 0.26$ & $0.87 / 0.26$ & $0.92 / 0.26$ & $0.97 / 0.24$ \\
1 & $0.48 / 0.17$ & $0.55 / 0.19$ & $0.62 / 0.22$ & $0.68 / 0.23$ & $0.74 / 0.24$ & $0.80 / 0.24$ & $0.85 / 0.24$ & $0.91 / 0.23$ & $0.96 / 0.21$ \\
2 & $0.44 / 0.15$ & $0.51 / 0.16$ & $0.58 / 0.17$ & $0.64 / 0.18$ & $0.70 / 0.19$ & $0.76 / 0.18$ & $0.82 / 0.17$ & $0.88 / 0.15$ & $0.94 / 0.11$ \\
3 & $0.41 / 0.09$ & $0.48 / 0.09$ & $0.54 / 0.098$ & $0.61 / 0.09$ & $0.67 / 0.075$ & $0.72 / 0.02$ & $\mathrm{~S}$ & $\mathrm{~S}$ & $\mathrm{~S}$ \\
4 & $\mathrm{~S}$ & $\mathrm{~S}$ & $\mathrm{~S}$ & $\mathrm{~S}$ & $\mathrm{~S}$ & $\mathrm{~S}$ & $\mathrm{~S}$ & $\mathrm{~S}$ & $\mathrm{~S}$ \\
\hline
\end{tabular}

(a)

(b)
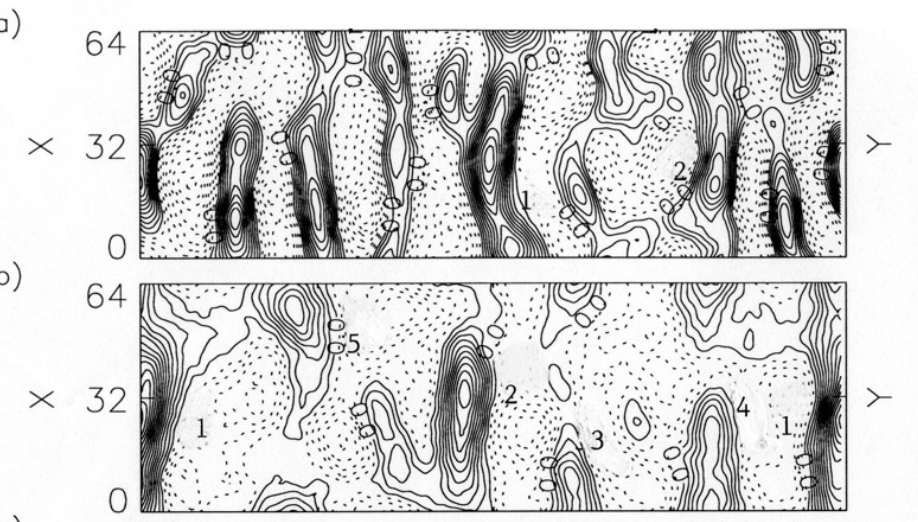

(c)

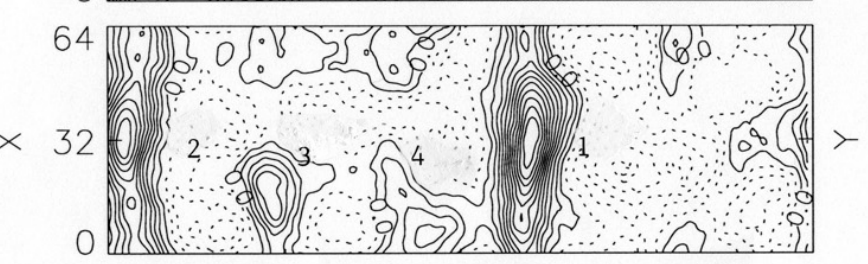

(d)

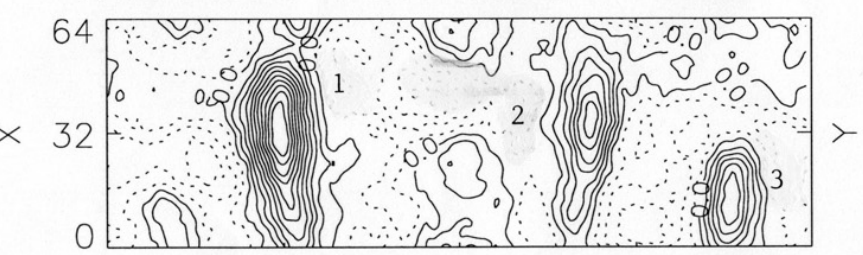

(e)

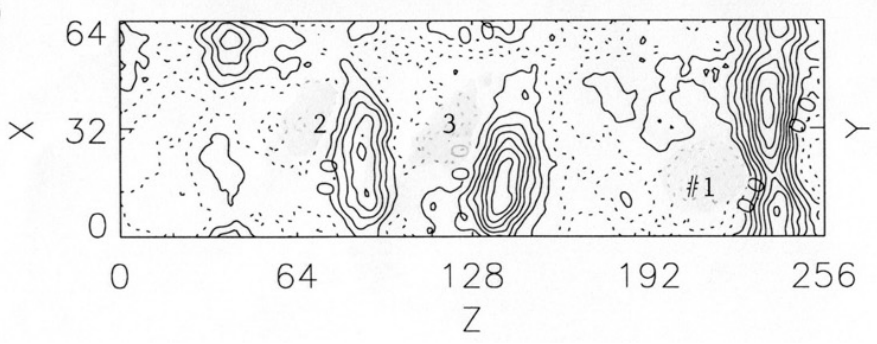

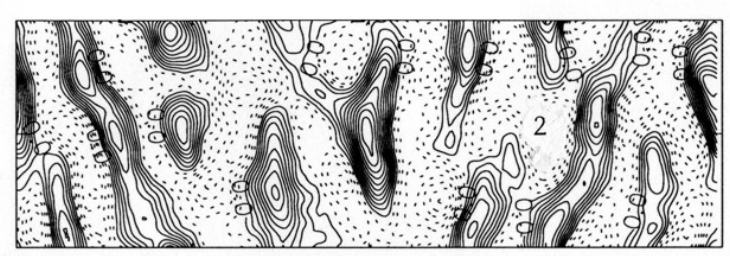
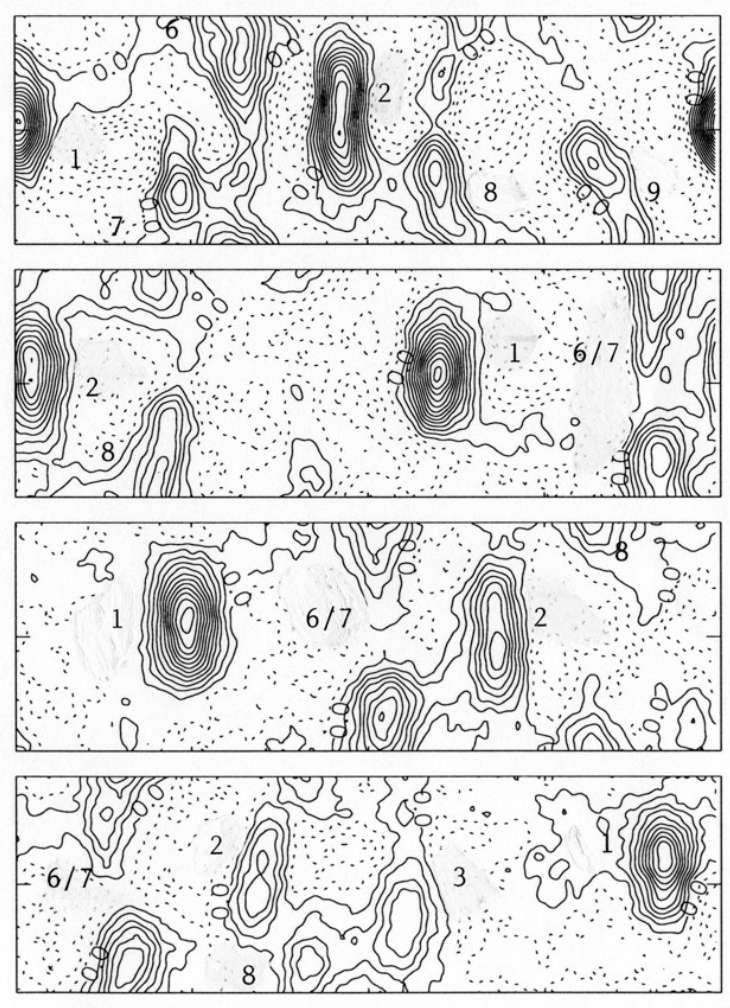

64

Fig. 5. Temporal evolution of the electron holes: (a)-(e) in the $x-z$ plane from $\bar{t}=100-500$ and (f)-(j) in the $y-z$ plane for the same times. Equipotential contours are plotted; the zero potential contour is labeled and the contour intervals are $\Delta \bar{\phi}=0.2$. Some of the potential pulses, which are electron holes, are numbered.

merged into one structure, which has a larger amplitude $\left(\sim 1.4 k_{B} T_{o} / e\right)$ compared to the amplitudes 0.6 for \# 2 and $1.2 k_{B} T_{o} / e$ for \# 3 before merging. After merging, we refer to the merged structure as \# 2 at later times. This is an example of merging of electron holes moving along the same magnetic field line. The structure \# 1 appears in Fig. 6a, but it is already developing undulations and perturbations in the equipotentials. The plots in the $y-z$ plane in panels (f) to (j) provide examples of interactions when transversely displaced electron holes come in close proximity to each other. Figure $6 \mathrm{f}$ shows that in the $y-z$ plane the merged structure \# 2 and \# 3 has pulled slightly ahead of \# 8 (see Fig. 5j). The 
(a)

(b)
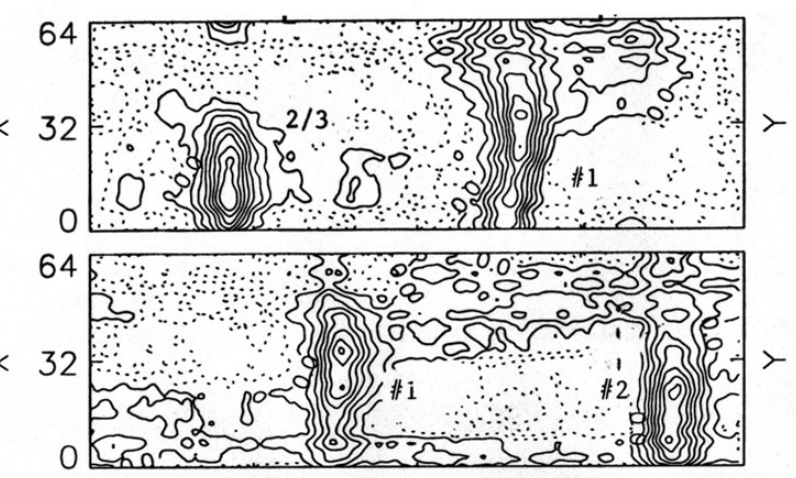

(c)

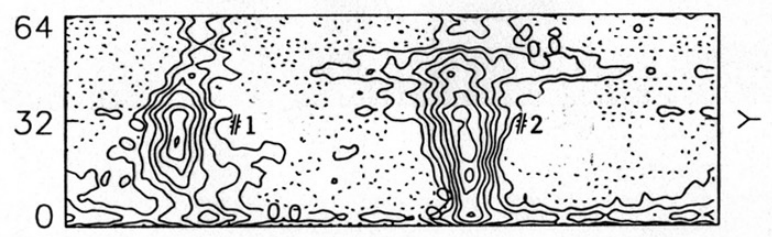

(d)

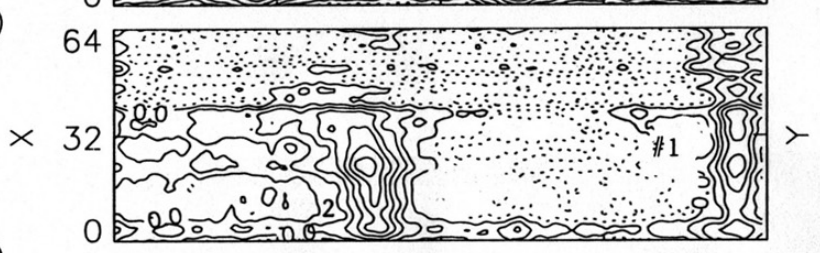

(e)

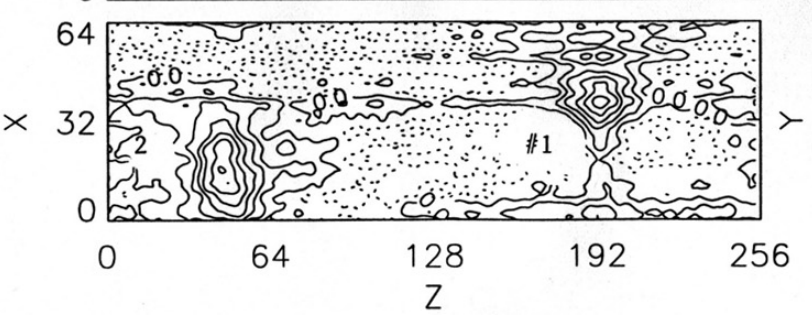

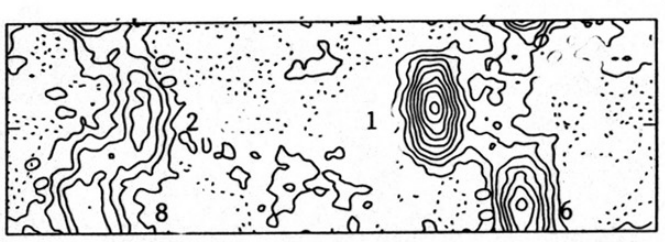

$(f)$

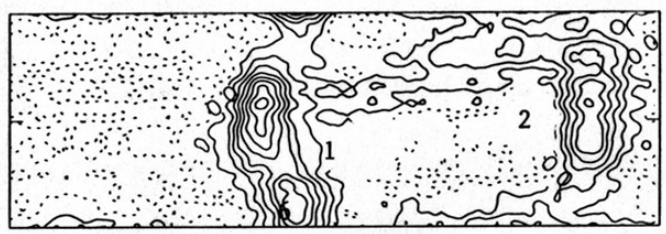

(g)

(h)
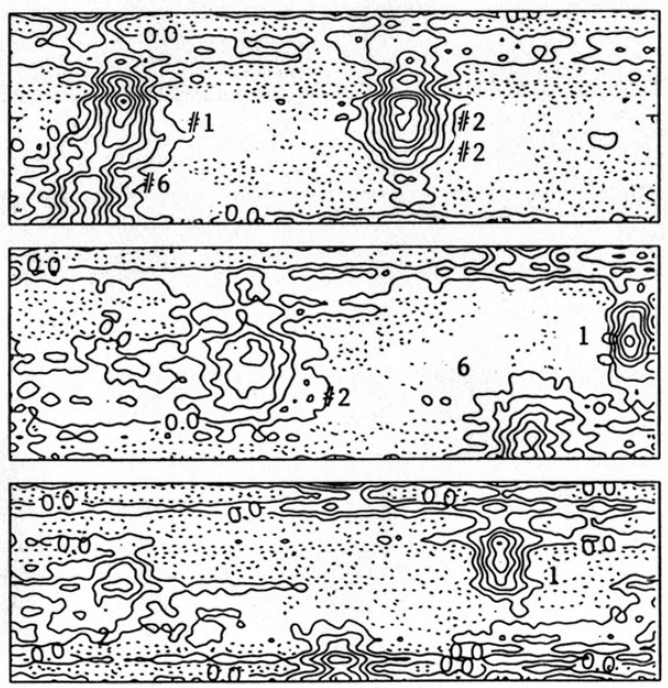

(j)

Fig. 6. Same as Fig. 5, but at later times from $\bar{t}=600-1000$. Note the decay of electron holes by emitting "fingers" of positive potential perturbations into the plasma.

merged structure \# 2 appears weaker in this plane compared to its amplitude in Fig. 5j. In this figure, we also see that \# 1 and 6 are quite close together. The panels $(\mathrm{g})$ to $(\mathrm{j})$ for later times show that \# 1 and 6 never merge together to become one structure; instead, we see that for $\bar{t}=800$ and 1000 structure \# 1 pulls ahead of \# 6 . In contrast to this behavior of \# 1 and 6, we find that \# 8 and 2 merge and become one structure at $\bar{t}=700$, and they remain so until the resulting structure decays. As a result of the merging, the amplitude of \# 2 increases from 0.6 at $\bar{t}=600$ to 1.2 at $\bar{t}=800$. This example shows that electron holes, which are displaced in directions transverse to $\boldsymbol{B}_{o}$, could merge when they come close to each other while moving parallel to the magnetic field. The evolution of the merging of the electron holes \# 2 and \# 8 in the $x-y$ plane is shown in Fig. 7. Figures $7 \mathrm{a}$ and $\mathrm{b}$ show the electron holes \# 8 and $2 \bar{t}=600$. \# 8 is centered at $\bar{z}=31$ and $\bar{y}=0$ or 64 , and it occupies the region $32<\bar{x}<64$ (Fig. 7a), while \#2 is centered at $\bar{z}=52$ and $\bar{y}=32$ and occupies $0 \leq \bar{x} \leq 32$ (Fig. 7b). The merged structures appear centered at $\overline{\bar{z}}=229$ and 155 at $\bar{t}=700$ and 800, as shown in Figs. 7c and d, respectively. The merged structures appear quite enlarged in the $x-y$ plane and are turbulent with undulations in the equipotential contours.

\subsection{Propagation velocity}

We find from the simulations that the velocity of an electron hole is not constant; it changes dynamically with the evolving plasma and other electron holes in its vicinity. The repeated appearances of the solitary pulses \# 1 and \# 2 in Fig. 1 b yield their velocities as a function of time. Furthermore, the distances traveled over each interval of time of duration $100 \omega_{p o}^{-1}$ determined from Figs. $5 \mathrm{a}-\mathrm{j}$ and $6 \mathrm{a}-\mathrm{j}$ could also yield the velocities. Table 2 shows the velocities determined by the latter method; these velocities agree well with the velocities determined by the repeated appearances. Initially, when the electron holes emerge from the oscillatory waves, the velocity of electron hole \# 1 is $\leq 4 V_{t e}$ and it remains so until $t \sim 200$. We recall that initial electron beam velocity is $4 V_{t e}$.

Table 2 shows the changes in the velocities of electron hole \# $1\left(V_{H 1}\right)$ and \#2 $\left(V_{H 2}\right)$. A noteworthy feature is that 

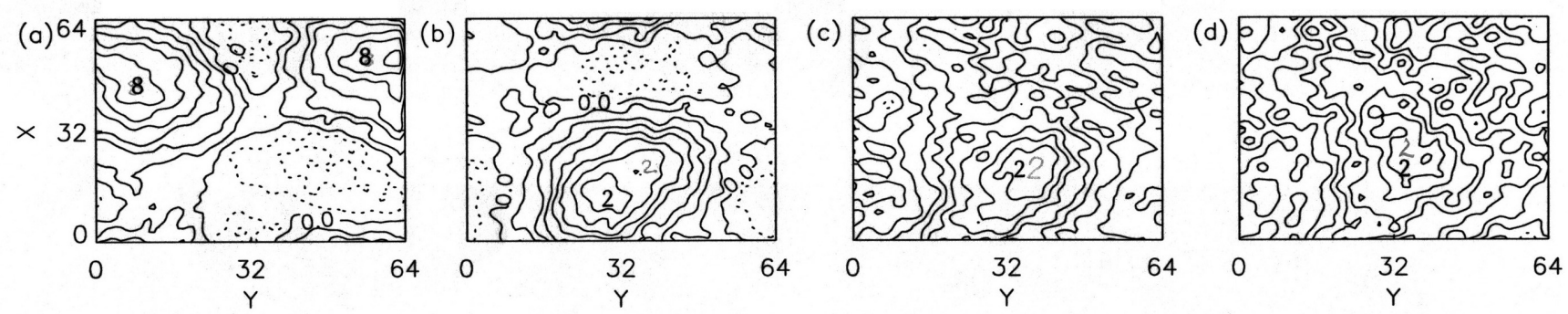

Fig. 7. Equipotential contours of electron holes \# 2 and \# 8 in the $x-y$ plane: (a)-(b) before merging at $\bar{t}=600$ and (c)-(d) after merging at $\bar{t}=700$ and 800 , respectively.

Table 2. Velocity of electron holes \# 1 and \#2 $\left(V_{H 2}\right)$ over intervals of time, each of $100 \omega_{\text {po }}^{-1}$

\begin{tabular}{llllllllll}
\hline $\bar{t} / 100$ & $1-2$ & $2-3$ & $3-4$ & $4-5$ & $5-6$ & $6-7$ & $7-8$ & $8-9$ & $9-10$ \\
$V_{H 1} / V_{t e}$ & $3 \cdot 9$ & $4 \cdot 1$ & $4 \cdot 2$ & $4 \cdot 3$ & $4 \cdot 4$ & $4 \cdot 4$ & $4 \cdot 6$ & $4 \cdot 6$ & $4 \cdot 6$ \\
$V_{H 2} / V_{t e}$ & $4 \cdot 2$ & $4 \cdot 0$ & $4 \cdot 2$ & $4 \cdot 2$ & $4 \cdot 8$ & $4 \cdot 3$ & $4 \cdot 4$ & $4 \cdot 5$ & $4 \cdot 6$ \\
\hline
\end{tabular}

when electron holes \#2 and \#3 merge during the time interval $500-600 \omega_{p o}^{-1}$, the velocity of the merged electron hole jumps to $4 V_{t e}$. Since electron holes are structures supported by a "hole" in phase space, the velocity of the potential structure is determined by the location of the hole in velocity space. Figures $8 \mathrm{a}$ and $\mathrm{c}$ show the phase space $\left(z-V_{z}\right)$ of the electron holes $\# 2$ and $\# 1$ at $\bar{t}=600$, respectively. The + signs show the holes in the phase space; their velocity location in phase space matches with the velocity of the potential structure given in Table 2. As expected, the hole corresponds to a minimum in the velocity distribution function $F\left(V_{z}\right)$. Figures $8 \mathrm{~b}$ and $\mathrm{d}$ show the corresponding distribution functions revealing the minima. The arrows point to the velocities of the electron holes tabulated in Table 2 for the time interval $500-600 \omega_{p o}^{-1}$. These figures also show that the electron holes are situated in the plateaued initial electron beam. The electron hole velocities evolve with the plateau. It is worth pointing out that in the process of the plateau formation, some of the beam electrons are accelerated beyond initial cold beam velocity, making it possible to have electron hole velocities larger than that for the initial beam.

Effects of electron holes on the plasma. We saw that by the time $\bar{t}=500$ in the simulations, the electron-hole potential structure begins to develop undulations and later on the undulations develop into long, thin "fingers" extending from the electron holes. This process leads to the decay of the electron holes (Singh et al., 2000, 2001a, b). A better way to further examine this decay process and its consequences is to look at the spatial structure of the transverse electric fields. Figures $9 \mathrm{a}$ and $\mathrm{b}$ show the structures in $E_{x}$ in the $x-z$ and $x-y$ planes, respectively, at $\bar{t}=1000$. The shades from dark to light in the gray scale plots represent from large, negative to large, positive values. $E_{y}$ shows similar structures. The noteworthy feature of the structure in $E_{x}$ in the $x-z$ and $x-y$ planes is that it has a long scale length along $z$ parallel to $\boldsymbol{B}_{o}$ and quite a short perpendicular wavelength $\lambda_{\perp} \sim \lambda_{x} \sim \lambda_{y}$ along the $x$ and $y$ directions. It is found that $\lambda_{\perp} \leq 10 \lambda_{d}$. For comparison, we note that in the simulation the average ion Larmor radius $\rho \cong 20 \lambda_{d}$. We have shown previously that such structures belong to the lower hybrid waves emitted by the fast moving electron holes (Singh et al., 2001a, b). These waves occur above the lower hybrid frequency given by $\omega_{\ell h} \cong \omega_{p t} /\left(1+\Omega_{e}^{2} / \omega_{p e}^{2}\right)^{1 / 2} \cong 0.021 \omega_{p o}$. Since the waves are electrostatic, they consist of density perturbations having structure similar to the structure in $E_{x}$ and $E_{y}$. Figure 10 shows the evolution in the density structure starting at $\bar{t}=200$ until the end of the simulation at $\bar{t}=1000$.

Figure 10a for $\bar{t}=200$ shows that there are randomly distributed fluctuations in the plasma which are quite faint. Formation of structures in the density begins to appear at $\bar{t}=400$ (Fig. 10b). However, the structures are still quite faint. For $\bar{t}>400$, as shown in Figs. 10c-e, there are welldeveloped structures corresponding to the developing fingers in the equipotential surfaces shown in Figs. 6a-e. These structures in the density distributions are like filaments that are long parallel to $\boldsymbol{B}_{o}$ and thin across it.

We have previously reported that when an initially fast filamentary electron beam drives a plasma, no electron hole structure forms. Instead, large-amplitude lower-hybrid waves and their decay into Alfvén resonance-cone waves at frequencies below the ion cyclotron frequency dominate the plasma (Singh et al., 2001c). Even in this case, the low-frequency waves below the ion cyclotron frequency accompany a filamentary density structure like that shown in Fig. 10. Therefore, we draw a larger conclusion here that whenever an electron beam drives a magnetized plasma the late-time behavior of the plasma results in the development of density structures, which are filamentary in nature.

Since the late time behavior in our simulation shows excitation of lower hybrid waves at frequencies $\omega \sim \omega_{\ell h}$, it is 

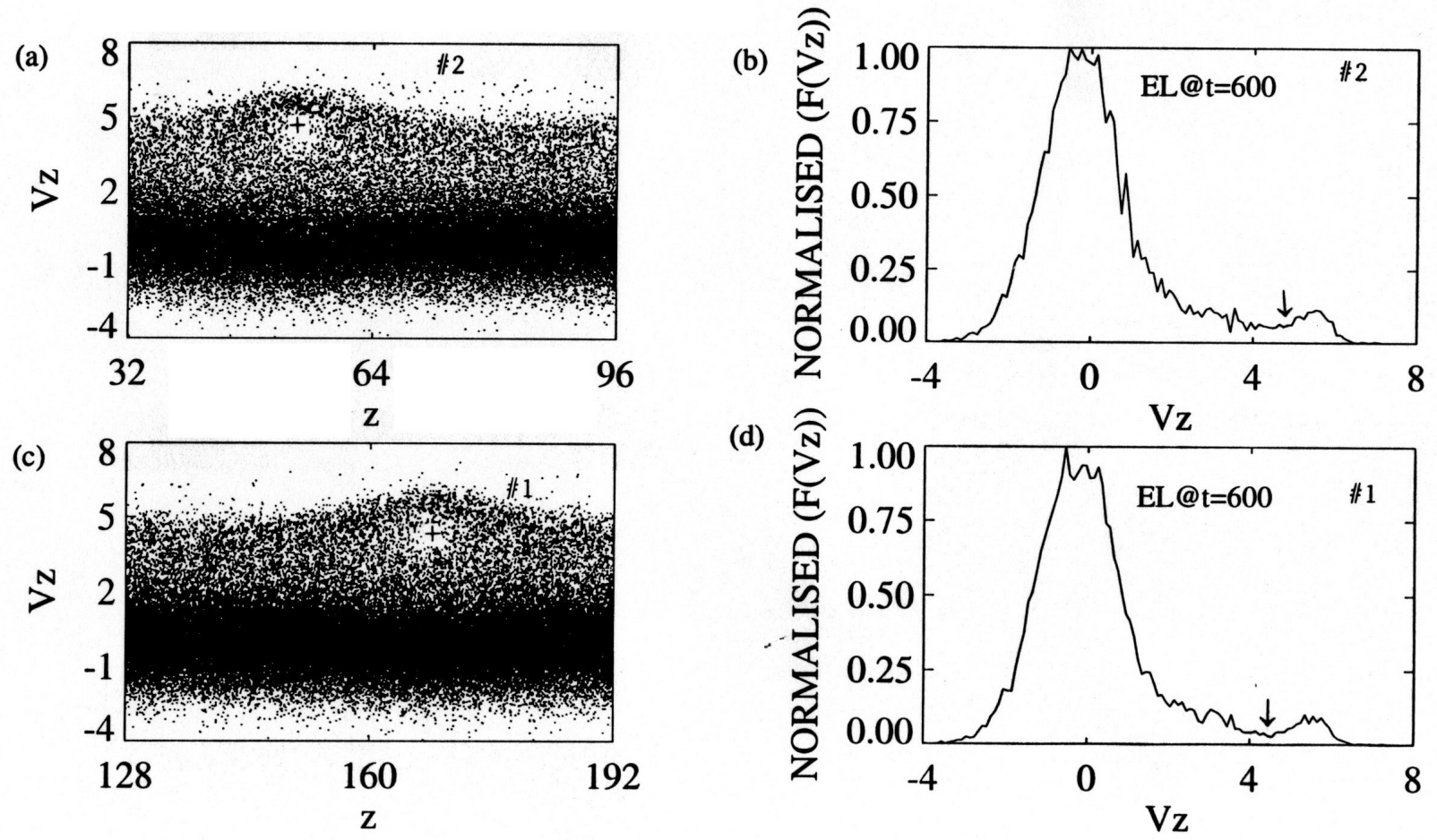

Fig. 8. Example of vortexes associated with the electron holes: (a) electron hole \# 2 at 600, (b) the electron velocity distribution associated with electron hole \# 2, (c) same as (a), but \# 1, and (d) same as (b), but for \# 1 .

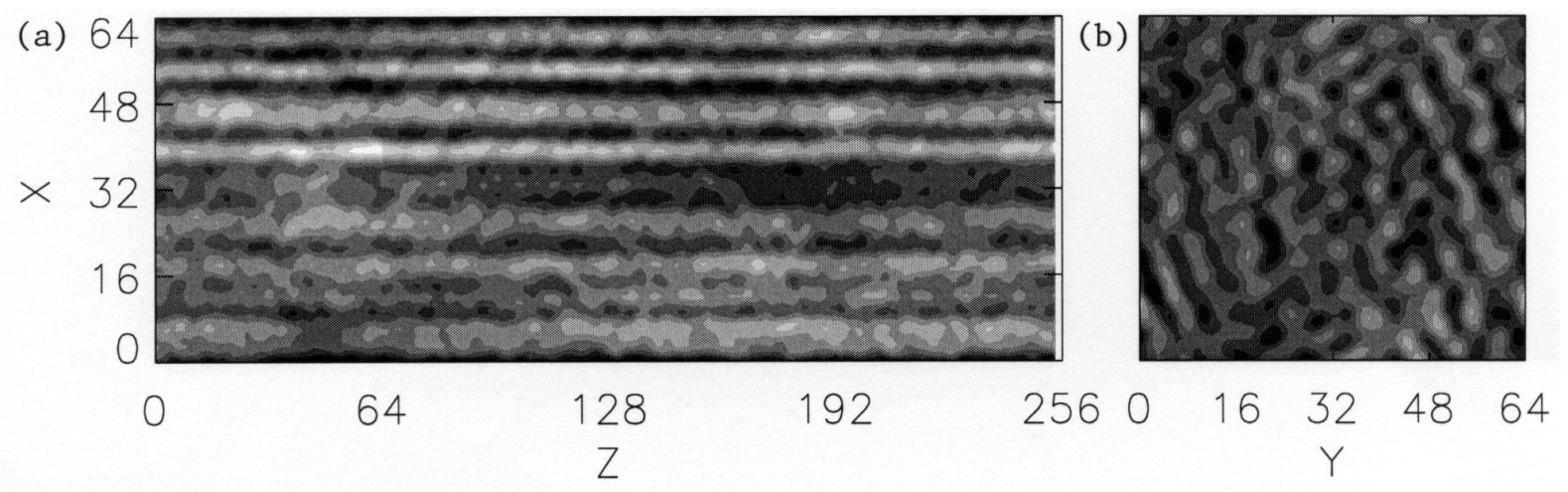

Fig. 9. (a) Wave structure of $E_{x}(x, z)$ at $\bar{t}=1000$ in the $x-z$ plane and (b) same as (a) but in the $x-y$ plane at $\bar{z}=128$. Note the long structures parallel to $z$ and they are periodic in $x$ and $y$ with periodicity length $\lambda \sim 10 \lambda_{d}$.

natural to ask whether or not these waves accelerate the ions transverse to $\boldsymbol{B}_{o}$. Figure 11a shows a typical example of the temporal behavior of the perpendicular electric field in the simulation box. The temporal features consist of the unipolar fields, as expected, from the passage of the electron holes and a relatively slow variation with a time period of the lower hybrid oscillations. The frequency spectrum of the fluctuations in Fig. 11a is plotted in Fig. 11b, showing that there is an enhanced power level in the spectrum for $0.02 \tilde{<} \omega / \omega_{p e} \leq 0.04$. What is the effect of such fluctuations on the ions?

Figure 12 shows the comparison of the ion velocity distribution function $F\left(V_{x}\right)$ at $\bar{t}=100$ and 1000 . We see that there is hardly any heating of the ions. $F\left(V_{y}\right)$ shows a similar feature as $F\left(V_{x}\right)$. The noteworthy modification in the transverse velocity distribution function, although not very dramatic, is near $V_{x}=V_{y}=0$, where the distribution is flattened somewhat. This is an indication of trapping of the low velocity ions in the potential structure associated with the electric fields shown in Figs. 9a and 11a. We calculated the average transverse energy of the ions at $\bar{t}=1000$ and found that $W_{\perp} \cong 1.01 k_{B} T_{o}$ compared to the initial energy of $1 k_{B} T_{o}$. What is the reason for the lack of the ion heating in the simulations?

The spatial structure of the waves shown in Fig. 9 showed 


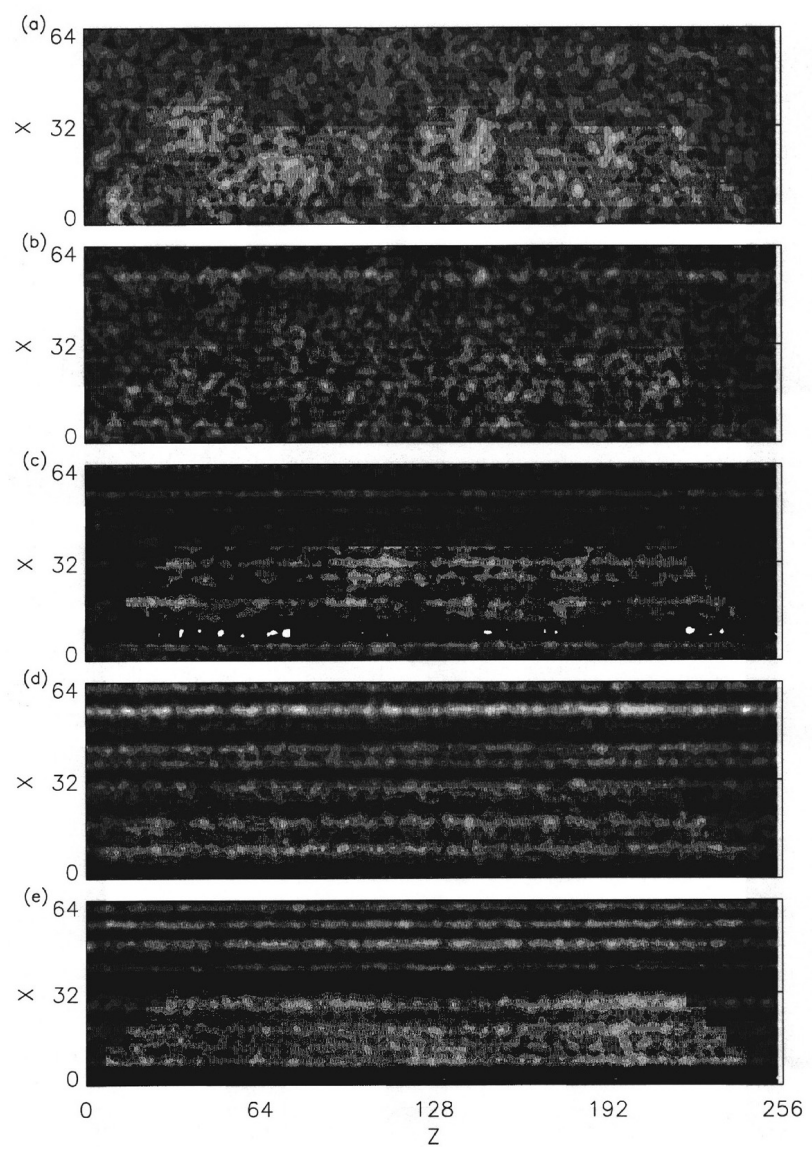

Fig. 10. Evolution of the density structure in the $x-z$ plane. The gray scale plots are at (a) $\bar{t}=200$, (b) $\bar{t}=400$, (c) $\bar{t}=600$, (d) $\bar{t}=800$, and (e) $\bar{t}=1000$. Note the formation of filamentary structures corresponding to the electric field structure in Figs. 9a and $b$.

that $\lambda_{\perp} \cong 10 \lambda_{d}$, which is nearly half of the average ion Larmor radius. The corresponding perpendicular phase velocity of the LH waves peaking above $\omega \cong \omega_{\ell h}$ is $V_{\perp p}=$ $\omega_{\ell h} / k_{\perp} \cong 0.02 \omega_{p e} \times 10 \lambda_{d} / 2 \pi \cong 0.03 V_{t e} \cong V_{t i}$. This shows that the short wavelength fluctuations could be in Landau resonance with the ions. This yields the trapping as mentioned above. Does the Landau resonance lead to transverse ion heating? If we assume that the heating is a random process, the heating rate could be shown to be

$\frac{\partial W_{\perp}}{\partial t}=1 / 2\left(e^{2} / M\right)\left|E_{\perp}\right|^{2} \tau_{c r}$,

where $\tau_{c r}$ is the correlation time of the fluctuations, and it is given as $\tau_{c r}=1 / \Delta \omega$, where $\Delta \omega$ is the bandwidth of the fluctuations. The Fourier spectrum in Fig. 11b shows that for the waves just above $\omega_{\ell h}, \Delta \omega$ is a few times $\omega_{\ell h}$, i.e. $\Delta \omega=\alpha \omega_{\ell h}$, where $\alpha$ is a factor of the order of unity. In terms of $W_{\perp}$ normalized in units of $k_{B} T_{o}$ and time in $\omega_{p o}^{-1}$, the heating rate becomes

$\partial \bar{W}_{\perp} / \partial \bar{t}=\frac{1}{2}\left(\frac{m_{e}}{M}\right)^{1 / 2} \frac{1}{\alpha}\left|\bar{E}_{\perp}\right|^{2}$.
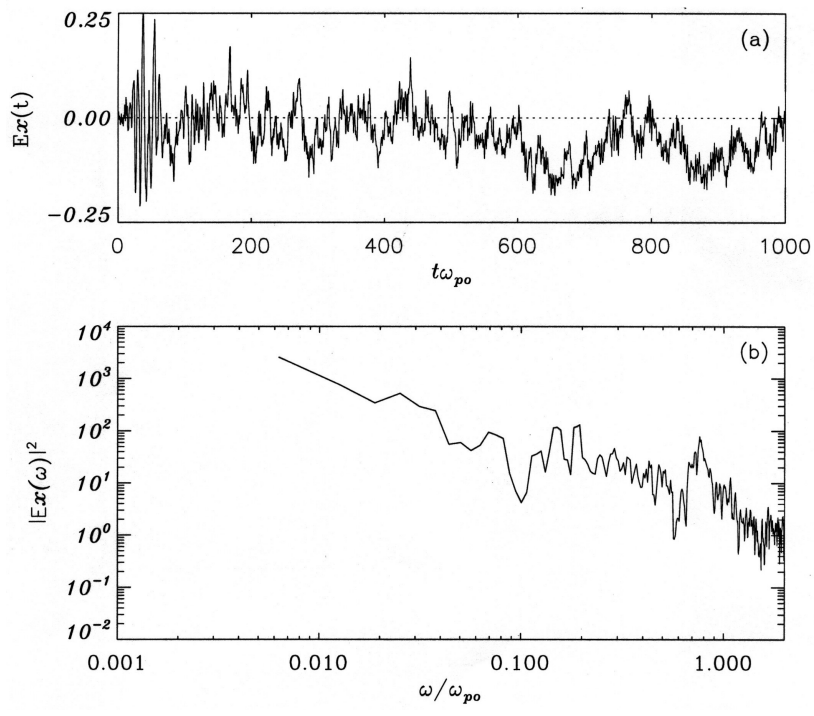

Fig. 11. (a) Temporal evolution of the transverse electric field $E_{x}(32,48,128)$ and (b) frequency spectrum of the data plotted in (a). Unipolar pulses belong to the electron hole \# 1 while the slow modulation is caused by the waves at $\omega \sim \omega_{\ell h} \cong 0.021 \omega_{\text {po }}$.

For the typical amplitude $\left|\bar{E}_{\perp}\right| \sim 0.1$, the normalized heating rate is $\sim 6 \times 10^{-5}$ for $\alpha=2$. Therefore, in order to see transverse ion heating by as small as $k_{B} T_{o}$, the simulation should run to about $16000 \omega_{p e}^{-1}$; it is difficult computationally to run the simulation for such a long time. We have run the simulation to only $1000 \omega_{p e}^{-1}$, thereby explaining the lack of transverse ion heating.

Let us apply these results of the simulations to estimate the time scale for ion heating in the auroral return current as reported by Ergun et al. (1998a, b) and Carlson et al. (1998a, b). If we assume $T_{o}=100 \mathrm{eV}$ and $\lambda_{d} \sim 100 \mathrm{~m}$ in a low density plasma, $\left|\bar{E}_{\perp}\right| \sim 0.1$ implies a strong field of $\sim 100 \mathrm{mV} / \mathrm{m}$, which is within the range of observed values. The heating time of $T>16000 \omega_{\text {po }}^{-1}$ implies a real time of $\sim 1 \mathrm{~s}$. This implies that the ion heating could be possible by the electron holes and associated plasma waves, if the ions interact over a time scale of a second or so, with a sustained high amplitude level of waves. This is possible only in the close vicinity of the diverging shocks in which electrons are accelerated upward, forming the beam. Fresh acceleration of electrons by double layers in the diverging shock maintains the high turbulence level needed for the heating (Singh, 2000).

In contrast to the lack of heating seen here, we have shown that when a filamentary electron beam drives large-amplitude lower hybrid waves, the ions are significantly heated by nonlinearly-driven stochasticity (Singh et al., 2001c). We also point out that, even though the electron holes have significant perpendicular electric fields in their structure, they do not affect the ions. 


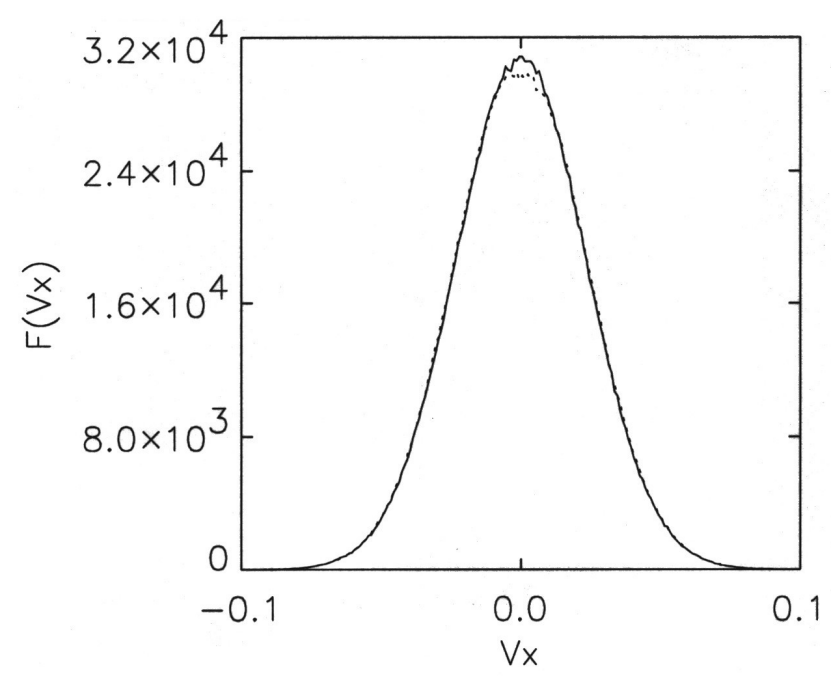

Fig. 12. Comparison of ion velocity distribution function $F\left(V_{x}\right)$ at $\bar{t}=100$ and 1000 . The plot at $\bar{t}=100$ is identical to the initial distribution. Note only a slight modification in the distribution near $V_{x} \sim 0$ due to the trapping of the ions in the wave field shown in Fig. 9. The parallel distribution $F\left(V_{z}\right)$ remains the same as the initial distribution and not shown here. The distribution $F\left(V_{y}\right)$ is identical to $F\left(V_{x}\right)$ shown here.

\section{Conclusion and discussion}

The main aim of this paper is to further examine some specific issues regarding the formation and dynamics of electron holes and their effects on the plasma when they are generated by electron-beam driven instabilities. For the first issue we have examined the emergence of electron holes of finite transverse sizes from oscillatory waves generated by the instability. Our conclusion here is that the beam generates resonantly high-frequency waves at frequencies $\omega<\omega_{p t}$, with $k_{\perp}<k_{\text {crit }}$, where $k_{\text {crit }}$ depends on the marginal stability criterion. In our simulation, the linearly generated waves have $k_{\perp} \leq m 2 \pi / L_{\perp}$ for $m \leq 3$ and $k_{\|} \cong n \times 2 \pi / L_{z}$ for $6 \leq n \leq 12$. We find that several of the modes have significant growth rates (see Table 1) in order to grow along with the dominant modes with $n \sim 11-12$ and $m=0$. The nonlinear interaction between the modes with $k_{\perp}=0$ and $k_{\perp} \neq 0$ leads to mutual modulation, resulting in the wave structure with finite transverse sizes. The nonlinear trapping of electrons in the crest of such a wave structure generates the electron holes having significant parallel $\left(E_{\|}\right)$and perpendicular $\left(E_{\perp}\right)$ electric fields from a very early stage of their formation and subsequent evolution.

In our simulations here, we have used $L_{x}=L_{y}=64 \lambda_{d}$. Therefore, the modes with $m \leq 3$ have transverse sizes $\ell \tilde{>} 20 \lambda_{d}$. In the return-current region of the auroral plasma $\lambda_{d} \sim 100 \mathrm{~m}$, yielding $\ell_{\perp} \sim 2 \mathrm{~km}$, which is an estimate for the perpendicular size of an electron hole. Our results here could be relevant to narrow diverging electrostatic shocks associated with return currents in which large-amplitude, nearly spheroidal electron holes were detected (Ergun et al., 1998a, b). The transverse scale of the shock structure affects the selection of $k_{\perp}$ spectrum.

The second issue we have discussed here is the evolution of electron holes from their birth from oscillatory waves until they nearly decay by emitting LH waves just above the lower hybrid frequency. We have shown the merging of electron holes, where merging occurs even when transversely displaced electron holes come into close proximity while propagating along the magnetic field. We have also examined the propagation velocity of the electron holes as the plasma and the electron holes evolve. We found that during the initial stage when electron holes have large amplitudes and a nearly laminar structure, their propagation velocity is slower than when the electron holes are highly perturbed and have grown weaker. This result is in contrast to the steady-state theory of Muschietti et al. (1999) in which the larger the amplitude of the electron holes is, the faster they move. Simulations show that the dynamic evolution of the plateaued electron beam determines the propagation of the electron holes.

The third issue we have examined here is that when electron holes decay by emitting LH or low-frequency electrostatic whistler (Singh et al., 2001a) waves, they cause perturbation in the plasma density. The perturbations are long structures parallel to the magnetic field, while they are quite narrow across it. This feature is quite similar to the density structure produced by parametric decay of large-amplitude lower hybrid waves (Singh et al., 1998, 2001c). In the simulation reported by Singh et al. (2001c), a fast electron beam of finite size across the ambient magnetic field did not generate electron holes; instead, large amplitude LH waves were generated. These waves decayed into Alfvén resonance-cone waves at frequencies below the ion cyclotron frequency. The density structures generated by these low-frequency waves were analogous to those reported here. Therefore, we draw a broader conclusion that when a magnetized plasma is driven by an electron beam which is slow or fast and has a small or large cross section across $\boldsymbol{B}_{o}$, the late stage of the evolution consists of density structures which are long filamentary structures.

We have also examined the question of transverse ion heating by the plasma waves emitted by the electron holes. In the simulations, we did not see any significant ion heating, but ion trapping in the waves by Landau resonances with the $\mathrm{LH}$ wave is apparent. The lack of significant heating is attributed to the length of time of the simulation. Estimates of the heating time is given, and it is pointed out that in the context of auroral return current plasma, the heating time is of the order of a few seconds.

Acknowledgements. This work was supported by NSF grant ATM98-14571. The author wishes to thank V. Ramanikumar for his help in preparing this paper.

\section{References}

Berk, H. L. and Roberts, K. V.: Nonlinear study of Vlasov's equation for a special class of distribution functions, Phys. Fluids, 10, 
1595, 1967.

Carlson, C. W., Pfaff, R. F., and Watzin, J. G.: The Fast Auroral SnapshoT (FAST) mission, Geophys. Res. Lett., 25, 2013, 1998a.

Carlson, C. W., McFadden, J. P., Ergun, R. E., Temerin, M., Peria, W., Mozer, F. S., Klumpar, D. M., Shelley, E. G., Peterson, W. K., Moebius, E., Elphic, R., Strangeway, R., Catell, C., and Pfaff, R.: FAST observations in the downward auroral current region: Energetic upgoing electron beams, parallel potential drops, and ion heating, Geophys. Res. Lett., 25, 2017, 1998 b.

Ergun, R. E., Carlson, C. W., McFadden, J. P., Mozer, F. S., Delroy, G. T., Peria, W., Chaston, C., Temerin, M., Elphic, R., Strangeway, R., Pfaff, R., Catell, C., Klumpar, D. M., Shelley, E. G., Peterson, W. K., Moebius, E., and Kistler, L.: FAST satellite observations of large-amplitude solitary structures, Geophys. Res. Lett., 25, 2041, 1998a.

Ergun, R. E., Carlson, C. W., McFadden, J. P., Mozer, F. S., Muschietti, L., and Roth, I.: Debye-scale plasma structures associated with magnetic-field-aligned electric fields, Phys. Rev. Lett., 81, 826, 1998 b.

Goldman, M. V., Oppenheim, M. M., and Newman, D. L.: Nonlinear two-stream instabilities as an explanation for auroral bipolar wave structures, Geophys. Res. Lett., 26, 1821, 1999.

Hubbard, R. F. and Joyce, G.: Simulation of auroral double layers, J. Geophys. Res., 84, 4297, 1979.

Loo, S. M., Wells, B. E., Singh, N., and Huang, E. P.: Case study: A portable parallel particle-in-cell code simulation, Proceedings of the International Conference on Parallel and Distributed Processing Techniques and Applications (PDPTA' 98), Las Vegas, Nevada, 13-16 July 1999.

Lynov, J. P., Michelsen, P., Pecseli, H. L., and Rasmussen, J. J.: Interaction between electron holes in a strongly magnetized plasma, Phys. Lett., 80A, 23-25, 1980.

Lynov, J. P., Michelsen, P., Pecseli, H. L., Rasmussen, J. J., Saeki, K., and Turikov, V.: Observations of solitary structures in a magnetized, plasma loaded waveguide, Phys. Scripta, 20, 328, 1979.

Matsumoto, H., Kojima, H., Miyatake, T., Omura, Y., Okada, M., Nagano, I., and Tsutsui, M.: Electrostatic Solitary Waves (ESW) in the magnetotail: BEN wave forms observed by GEOTAIL, Geophys. Res. Lett., 21, 2915, 1994.

Miyake, T., Omura, Y., Matsumoto, H., and Kojima, H.: Twodimensional computer simulations of electrostatic solitary waves observed by Geotail spacecraft, J. Geophys. Res., 103, 11841 , 1998.

Mozer, F. S., Ergun, R., Temerin, M., Cantell, C., Dombeck, J., and Wygant, J.: New features of time domain electric field structures in the auroral acceleration region, Phys. Rev. Lett., 79, 1281,
1997.

Muschietti, L., Ergun, R. E., Roth, I., and Carlson, C. W.: Phasespace electron holes along magnetic field lines, Geophys. Res. Lett., 26, 1093, 1999.

Muschietti, L., Roth, I., Carlson, C. W., and Ergun, R. E.: Transverse instability of magnetized electron holes, Phys. Rev. Lett., 85, 94, 2000.

Omura, Y., Matsumoto, H., Miyake, T., and Kojima, H.: Electron beam instabilities as generation mechanism of electrostatic solitary waves in the magnetotail, J. Geophys. Res., 101, 2685, 1996.

Omura, Y., Kojima, H., Miki, N., Mukai, T., Matsumoto, H., and Anderson, R.: Electrostatic solitary waves carried by diffused electron beams observed by the Geotail spacecraft, J. Geophys., Res., 104, 14 627, 1999.

Oppenheim, M. M., Goldman, M. V., and Newman, D. L.: Evolution of electron phase-space holes in 2-D magnetized plasma, Phys. Rev. Lett., 73, 2344, 1999.

Saeki, L., Michelsen, P., Pecseli, H. L., and Rasmussen, J. J.: Formation and coalescence of electron solitary holes, Phys. Rev. Lett. 42, 501, 1979.

Schamell, H.: Kinetic theory of phase space vortices and double layers, Phys. Scr. I, 2/1, 228, 1982.

Singh, N.: Computer experiments on the formation and dynamics of electric double layers, Plasma Physics, 22, 1, 1980.

Singh, N.: Double layer formation, Plasma Phys., 24, 639, 1982.

Singh, N.: Electron holes as a common feature of double-layerdriven plasma waves, Geophys. Res. Lett., 27, 927, 2000.

Singh, N. and Schunk, R. W.: Plasma response to the injection of an electron beam, Plasma Phys. Controlled Fusion, 26, 859, 1984.

Singh, N., Loo, S. M., Wells, B. E., and Deverapalli, C.: Threedimensional structure of electron holes driven by an electron beam, Geophys. Res. Lett., 27, 2469, 2000.

Singh, N., Loo, S. M., and Wells, B. E.: Electron hole as an antenna radiating plasma waves, Geophys. Res. Lett., 28, 1371, $2001 \mathrm{a}$.

Singh, N., Loo, S. M., and Wells, B. E.: Electron hole structure depending on plasma magnetization, J. Geophys. Res., 106, 21 183-21 198, 2001b.

Singh, N., Loo, S. M., Wells, B. E., and Lahkina, G. S.: Evolution of electron beam generated waves resulting into transverse ion heating and filamentation of the plasma, J. Geophys. Res., 106, 21 165-21 182, 2001c.

Singh, N., Wells, B. E., Abdelrazek, A., Al-Sharaeh, S., and Leung, W. C.: Three dimensional kinetic simulation of the nonlinear evolution of lower hybrid pump waves, J. Geophys. Res. 103, 9333-9349, 1998.

Turikov, V.: Electron phase space holes as localized BGK solutions, Phys. Scripta, 30, 73, 1984. 